\title{
The Status of Women in Ainkurunuru Palai Thinai
}

\section{ஐங்குறுநூறு பாலைத்திணையில் மகளிர் நிலை}

\section{J. Jayavanisri a, *}

a Department of Tamil, Government Arts College (Autonomous), Kumbakonam-612002, Tamil Nadu, India.

*Corresponding author Email: jayavanisrijayapal@gmail.com

DOI: https://doi.org/10.54392/ijmrd2211

Received: 17-11-2021; Revised: 29-01-2022; Accepted: 31-01-2022; Published: 04-02-2022

Abstract: The mind of the leader is multifaceted. Her mindset is to lose all of her thoughts, flow, thought, action so that she regrets it and then regains what she lost and then rejoices. Without the leader the leader has no responsibilities. Leader Semmandu is aiming to join hands with the leader's love. The mood of the leader is calmed by the word of the friend. Without the support of a friend, the life of the leader will be in question. The reason why the leader excels in the inner mantra is the fear, madness, shame and cultivation she finds. The nurse regrets not thinking of her son's life and thinking of Nartra's daughter. The nurse also appears to be the real mother to the leader. The nurse understands the mother's instincts and actions and is a mother in the internal literature who participates in the suffering of the leader who appears to be the mother.

Keywords: Ainkurunuru, Palai thinai, Ancient Tamil People, Palai Culture

Language: Tamil

\section{முன்னுரை}

ஐங்குறுநூற்றில் ஒவ்வொன்றுக்கும், குறைந்த அடி வரையறை பெற்ற ஐங்குறுநூறு பாடல்களைத் தனித்தனியாகப் பெற்றுள்ளமையால் ஐங்குறுநூறு எனும் நூல் அப்பெயரினைப் பெற்றுள்ளது. ஐங்குறுநூற்றுப் பாலைத்திணைப் பாடல்களில் ஓதலாந்தையாரின் உணர்வு நிலையைக் காட்டுதல் பயனுடையது என்று கருதி இக்கட்டுரை அமைகிறது.

தலைவன் எப்போதும் பிரிய வேண்டும் என்கிற எண்ணம் இல்லாதவன். காலம் அறிந்து பிரிய நினைக்காமல் பிரியும் தலைவன் மதிகெட்டவனாக அறியபடுவான். தோழியிடம் தலைவி புலம்புவாள். மழைபெய்து இலை உதிர்த்த மரமெல்லாம் பூத்து குலுங்க ஆரம்பித்துவிட்டது. அதுமட்டுமல்ல இளைய காதலர்கள் பிரிய முடியாத கனமான இலவ மரம் பூக்கும் காலத்தின் தலைவன் பிரிந்தான் (Parimanam \& Balasubramaniyam, 2004).

தலைவன் பிரிந்து வெகுகாலம் ஆனாலும் உரிய பருவத்தில் தலைவன் வரவில்லை என்றால் அவள்படும் துன்பம் தான். அதுவும் மாலைநேரம் அஃறிணைப் பொருட்களின் கூடலை பார்த்தால் பருவத்தின் காத்திருப்பு ேேதனையை ஏற்படுத்தும். உரிய பருவத்தில் வராத தலைவன், "அம்மவாழி தோழி சிறியினை" என தலைவி புலம்பும் நிலையை காட்டுகின்றார் (Parimanam \& Balasubramaniyam, 2004).

\section{இளவேனில் பருவமும் துன்பமும்}

தலைவன் இளவேனில் பருவம் வந்ததும் வருவதாகச் சொல்லிப் பொருள் தேடிப் பிரிவான். தலைவி அப்பருவம் வரக் காத்திருப்பாள். குறித்த நேரம் வராமையால் வருத்தம் மிகுந்து துன்பத்தில் புலம்புகிறாள். தலைவியின் கூந்தல் மலர் சூடாமல் இளவேனிற் பருவத்தைக் கண்டு கண்ணீர் சிந்துகிறாள். அதற்குக் காரணம் காதல் உணர்ச்சியை தூண்டும் போது தலைவன் வரவில்லையே என்று 
தலைவி, “அவரோ வாரார்" பூக்கள் பூக்கும்போது அதைக்கண்டு பறவைகள் கத்தும் போது தலைவன் வராததை எண்ணி புலம்பியும், இங்கு கூவும் குயில் தலைவியின் பிரிவால் குயில் பிரிந்தது போல பிரிந்த தலைவனை ஞாபகப்படுத்தியும் குயில் கூவும் என்பதை, “அஞ்சினைப் பாதிரி” என்று கூறுகின்றார் (Parimanam \& Balasubramaniyam, 2004).

தன்னை ஒருமுறை கூடிய பொழுது தலைவன் என்னை மட்டும் உயிர் என்றான். நான் இப்போது தாய்மை நிலையில் உள்ளதால் வேறு பெண்ணை தலைவன் நாடியதை, "முளை நிறை முறுவல்” (369) என்பதில் அலர் என்னை கொள்கிறது என்றாள். பரத்தையோடு கூடியதை பொய்யுரைத்த தலைவி மனதில் ஆயிரம் ஏக்கத்தோடு அவளோடு சேர்ந்து வாழ்க என்பதை, “நீ நயந்து உறைய|| (370) என்றாள். உடன்போக்கு மேற்கொண்ட தலைவி தலைவனோடு தன் அழகு கெடாமல்; பாலைவழியைக் கடந்து வருகிறாள். செவிலி வளர்த்த அழகு நான் வருவதாக கூறுங்கள் என்று பார்ப்பனர்களிடம் கூறுகிறாள். தாய்நயந்து எடுத்த ஆய்நலம்" எனும் பாடலில் தலைவியின் இன்பவுணர்வு வெளிப்படுகிறது. பெற்றோர் தலைவியைத் தலைவனுக்குத் திருமணம் செய்ய மறுத்தனர். தலைவனுடன் உடன்போக்கு சென்ற தலைவி, இடையில் காண்டாரைப் பார்த்து என் தாய்க்கு நீங்கள் “கடும்கண் காணயொடு நெடுந்தேர்” என் தாயிடம் உரைக்குமாறு அமைந்துள்ளு. தோழி தலைவியின் மேனி அழகு கெட்டதை எண்ணி புலம்புகிறாள். தோழியே நீ வருந்த வேண்டாம். வருந்தினால் நான் மீண்டும் எனக்கு மகிழ்வை நினைத்த தலைவி “எல்லையில் இடும்பை தரூஉம்” என தலைவியின் துன்பம் மறந்த இன்ப நிலையை அறியமுடியும் (Parimanam \& Balasubramaniyam, 2004).

\section{தோழியின் மனநிலை}

தோழி இல்லையேல் தலைவன், தலைவி இருவருக்கும் களவு, கற்பு, என்பது இல்லாமல் போகும். தோழி ஒவ்வொரு நிலையிலும் தலைவிக்கு அரனாக செயல்படுபவள். அதுமட்டுமல்லாமல் அடைப்பட்ட நீர் வெளிவர அணையின் மதகு உதவுவது போல, தோழி தலைவியின் மனதிற்கு ஒரு நல்ல மதகாக இருப்பாள். தலைவியின் மனம் வருத்தும் பொதெல்லாம் அவள் மனத்திற்கு ஒரு மனமாகவே தோழி செயல்படுவாள். தலைவன், தலைவி இருவரின் ஒவ்வொரு செயலும் தோழிக்கு தெரியாமல் நிகழாது. அவன் இல்லையேல் ஒரு அணுவும் அசையாது என்பது போல் தோழி இல்லையேல் தலைவன், தலைவியின் காதல் உயிர் பெறாது என்பதை உணரலாம்.

\section{சுரத்தருமை கூறிய தோழி}

கோடைக் கொடுமைக்கு ஆற்றாது வரும் பறவைகளுக்கு ஆலமரம் குளிர்ந்த நிழலும், இனிமையான கனியும் கொடுத்து உதவும். அதுபோல தலைவிக்கு தலைவனின் சேர்க்கையிலும் அவன் உடன் இருப்பதாலும் வழியின் கொடுமை தெரியாது என்றும் பிரிய நினைக்கும் தலைவனிடம் எங்களையும் அழைத்து செல்வாயாக என்கிறாள். "தண்ணிய இனிய" எனும் பாடலில் தலைவனிடம் தோழி, இனி நொடி பொழுதும் உன்னை விட்டு பிரிய நினைக்க மாட்டாள். நீ பிரிந்தால் அவள் அழகு கெடும் என்பதை கூறுகிறாள். செல்லாய் ஆயினோ" என்பதில் காடும், மலையும் ஆபத்தான இடங்கள் என்று தலைவிக்குச் சொல்ல தேவையில்லை. பாதுகாப்பில்லாத காட்டை, "மைவரை நாட வருந்துவள் பெரிதே" எனக் குறிப்பிடுவதால் விளங்கும் (Parimanam \& Balasubramaniyam, 2004).

\section{தோழியின் வாய்மை}

தலைவன் பொருள் வரைவின் பிரிவதை தோழி, இவ்வளவு நாள் தலைவியோடு தனிமையில் பல இடங்களில் பழகினாய். இப்போது நீ பிரிந்தால் தலைவியின் தனிமைத் துயர் அவளை பசலையில் ஆட்படுத்தும் என்பதை, "புயல் நெருங்" வெளிப்படுத்துகிறாள். தலைவன் தான் மேற்கொண்ட காரியம் கைகூட புறப்படுகின்றான். அப்பொழுது அவன் செல்லும் வழியில் உணவின்றிப் பசியால் 
வருந்தினாலும் பிடியைப் பிரியாத பாடல் களிறு உடன் தழுவி நிற்கும். இதனை, “களிறு பிடிதழீஇப் பிறபுலம்” எனத் தோழி தெளிவாக்குகிறாள் (Parimanam \& Balasubramaniyam, 2004).

\section{பொறுத்துக் கொள்ளாத துயரம்}

தலைவன் கடுமையான ஆபத்து தரும் காட்டுவழியில் செல்லுகிறான். அவன் செல்லும் வழியில் கொடிய புலிகள் திரிவதை, பொருள் தேடும் விருப்பத்தில் இருக்கும் தலைவன் காட்டின் தனிடையில் நம்மை மறந்து இப்பெரும் துயரத்தை எங்ஙனம் பொறுப்பது என்பதை, "பிரிதல் உலகுவை " எனும் பாடல் வழி தோழி கூறுகிறாள். தலைவனின் வரவை உணர்ந்த தோழி, தலைவன் எடுத்த காரியத்தை உடனே முடிக்கும் திறன் பெற்றதால் விரைந்து வந்தார் என்பதை, "பெருங்கை யானை" எனும் பாடலில் விரிக்கிறார். தலைவன் வரும்வழி சிறிதும் பாதுகாப்பு இல்லாதது. இருப்பினும் அருமையான உன் பண்பின் நினைவில் விரைந்து வருவார் என்பதை, "அரிய சுரன் வந்தனரே" எனக் குறிக்கின்றாள் (Sivalingan, 1995).

\section{அறத்தோடு நிற்றல்}

தலைவி தலைவன் மீது கொண்ட காதல் மிகுதியால் உடல் மெலிந்து காணப்படுகிறாள். அதனைக் கண்ட செவிலி வினவ, தோழி தலைவியின் களவை வெளிப்படுத்துகிறாள் "அறிய ஆகுமோ மற்றே” (366) என்னும் பாடலால் கூறி, தலைவியை பெண் கேட்டு அமலயார் வந்தபோது தலைவியின் இதயம் நொறுங்குகிறது. இதனைக் கண்ட தோழி, வேனில் பருவத்தில் மலரும் பல்வேறு மாலை பறிப்பதற்காகத் தலைவி காட்டாறுப் பக்கம் சென்றாள். நீரில் மிதந்து வரும் பூக்களை எடுக்க முயன்றபோது தலைவி நீரில் விழுந்தாள். செல்வனாகிய தலைவன் உடனே தேரில் வந்து ஆற்றில் விழுந்து தூக்கி அவளுக்கு உயிர் தந்தான். அவளுடைய உயிர் அவனுடைய பெயரோடு புணர்ந்திருக்கிறது என்று தோழி பூத்தரு புணர்ச்சியை, "விரவுமலர் அணிந்த" என உணர்த்தும் பாடலில் களவொழுக்கத்தைச் செவிலிக்கு உணர்த்துகிறாள் (Sivalingan, 1995; Ilankumaranar, 2018).

தலைவன் உரிய காலத்தில் தலைவியிடம் தான் குறித்த காலத்தில் வருவேன் என்று பிரிகிறான். ஆனால் தோழி அதுவரை உயிர் வாழ்ந்தால் இன்பம் நுகர்க, “எம்மோடு கொண்மோ" (368) என கூறுவதோடு, “நினைத்தொறும் கவிழும்" என தலைவி தலைவனோடு உடன்போக்கு சென்றால் ஊரார் அவள் தூற்றியதை தான் பார்த்ததும், கேள்வியுற்றதையும், ஊரார் வருத்தியதையும் எடுத்துரைக்கின்றாள்.

\section{நற்றாயின் ஆதங்க புலம்பல்}

இவ்வுலகில் உயர்திணை ஆயினும் அஃறிணை ஆயினும் தாயே முதன்மையாக விளங்குகிறாள். பெண்ணாகிய தாய் இல்லையென்றால் உயிரின் இயக்கம் இல்லை. தாயின் அன்பை விளக்க வந்த தொல்காப்பியர்,

‘தாய்போல் கழறீத தழீ இயக் கோடல்

ஆய்வினை கழத்துக்கும் உரித்தென"

என்கிறார் (Sivalingan, 1995). சங்க இலக்கிய அகப்பாடல்களில் தாயுறவு நற்றாய், செவிலித்தாய் ஆகிய இருநிலைகளில் அமைகின்றன.

உடன் போக்கு அறநெறி எனத் தெளிதல் 
நற்றாய் தம் மகள் உடன்போக்கில் சென்ற நிலையில் பலவாறு வருந்துகிறாள். இறுதியில் தலைவியின் முடிவை ஏற்கனவே செய்கிறாள். தலைவி களவொழுக்கக் காலத்தில் தனக்குரியவனாக ஏற்றுக் கொண்ட தலைவனுடன் களவொழுக்கம் பூண்டு வாழவே சென்றுள்ளாள் என்ற நிலையில்,

"மன்னர் கொட்டின் மஞ்ஞ ஆலும்

உயர்நெடும் குன்றம் படுமழை தழீஇச்

சுரம்நனி இனிய ஆகுக தில்ல

அறநெறி இதுவெனத் தெளிந்த என்

பிறைநுதல் குறுமகள் போகிய கான"

என்று தன் மனதை தேற்றிக் கொள்கிறாள். பண்டைத் தமிழர் களவொழுக்கமாக, அச்செயலை அக இலக்கியத்துறையில் வாழ்வியல் நெறியாக அமைந்துள்ளது புலனாகிறது (Parimanam \&

Balasubramaniyam, 2004).

\section{மனம் புலம்பல்}

தன்செயல் எதுவானாலும் செய்து முடிக்கத் துடிக்கும் தலைவியின் குணம் ஆழமானது. அவள் தன் தலைவனோடு உடன்போக்கு மேற்கொண்டாலும் பின்வருவதை அறியாத பதுமையாக தலைவனின் கரத்தைப் பிடிக்கின்றாள். நெருப்பின் அருகே வைத்த காகிதம் போல் தலைவி உடன் போக்கை வைத்து புறப்பட்டாள். அவள் தூற்றும் சமூகத்தில் என்னையும் நினைத்துப் பார்த்தாள் என்று நற்றாய் புலம்புகிறாள். "என்னும் உள்ளினள்" எனும் பாடலில் மனம் புலம்புவது வெளிப்படுகிறது (Parimanam \& Balasubramaniyam, 2004).

\section{தலைவன் தாயைச் சபித்தல்}

நற்றாய் தன் மகள் இல்லாத வீட்டைக் கண்டு கலங்கும் நிலையில் அதற்குக் காரணமான தலைவனை வெறுக்கிறாள். அவள் தான் தன் மகளைப் பிரிந்து வருந்துவதைப் போலத் தலைவனுடைய தாய் அவளுடைய மகனைப் பிரிந்து வாட வேண்டும் என்பதை, “நினைத்தொறுங் கலிலும்" ஆம் பாடலில் குறிப்பிட்டு காட்டுகிறார் ஆசிரியர் (Parimanam \& Balasubramaniyam, 2004).

\section{தலைவியின் இளமையை கூறிய நற்றாய்}

“காக்கைக்கு தன் குஞ்சு பொன் குஞ்சு" என்பது போல தலைவியின் ஒவ்வொரு செயலும் நற்றாய்க்கு அழகைத் தருவதாக அமையும். தலைவியின் ஒவ்வொரு பருவத்திலும் வளர்த்த நற்றாய் அவளின் இளமை தன்மையை பெரிதும் நினைத்து கவலை கொள்வாள். அவள் இளமை பருவத்தில் காதலில் அகப்பட்டு தலைவனோடு உறவுகளை துறந்து புறப்பட்டதை நினைத்து நற்றாய் வருந்துகிறாள். காப்பையும் பொருட்படுத்தாமல் தலைவி சென்றதை நினைத்து அவளின் இளமையின் அழகை நினைத்து நற்றாய் இரங்கிக் கூறுகிறாள் (Parimanam \& Balasubramaniyam, 2004).

தலைவியை உடன்போக்கில் அழைத்துச் சென்ற தலைவனை தாய் திட்டுகிறாள். பின் தலைவியை இழந்தும், பிரிந்தும் மனவேதனை அடைந்து நற்றாய் தலைவனின் தாய் பழிப்பதாக, “பூங்கண் ஆயம்" அமைந்துள்ளது. இவ்விரு கூறுகளும் தலைவியின் தாய் என்னைப் போலவே மனவேதனை படவேண்டும் என்ற பொருளில், "பல லூழ் நினைப்பினும்" என்று காட்டுவது நோக்கத்தக்கதாகும் (Parimanam \& Balasubramaniyam, 2004). 
காண்போரிடம் கூறுதல்

நற்றாய் தன் மகன் இல்லாத சூழலில் அவள் பயன் படுத்திய அத்துணைப் பொருட்களையும் பார்த்துப் பார்த்து உள்ளடங்குவதை, இது என் பாவை என்ற பாடலில் தன் மகளுக்குச் சொந்தமாக இரந்த பாவையெங்கில், பூவை முதலியவற்றை கண்டு ஏங்கும் தாயுள்ளத்தைச் சித்தரிக்கின்றது. மேலும், "மணிவாழ் பாவை" எனும் பாடலில் நற்றாய் தலைவியை பிரிந்து அவள் பயன்படுத்திய ஓவ்வொரு பொருளையும் பார்த்து புலம்பவதைக் கூறுகிறது (Dakshinamurthy, 2004a).

\section{விதியை சபித்தல்}

ஒரு தாய் அவள் மகளை வளர்க்கும் துயரம் வறுமையின் பீடி இன்னப்பிற துன்பங்கள் வந்தாலும் தலைவியை கைவிடமாட்டாள். அதுபோல தன் தலைவி பிரிவுக்கு காரணமான ஒவ்வொரு பொருளையும் நற்றாய் சபிப்பது வழக்கமான செயலாகும். ஆனால் பெற்றதாய் விதியை சபிக்கின்றாள். “நாடெனும் கலிழும்" எனும் பாடலில் நற்றாய் உள்ளம் சபித்தாலும் நிறம் மாறாத பாலாகவே எப்பொழுதும் இருக்கும். அவள் மனமும், நினைப்பும் உடன்போக்கு மேற்கொண்ட தலைவி கடக்கும் ஒவ்வொரு பொழுதையும் எண்ணி முதலில் வருந்துபவள் தாயாகத்தான் இருப்பாள். வழியின் தன்மை நிகழும் செயல்கள் அனைத்தையும் பார்த்தது போல அறியும் புத்தி படைத்தவளாக நற்றாய் விளங்குகிறாள். தலைவியின் நிலையுணர்ந்த தாய் அவளை நினைத்தே விழிகளும் உறங்காமல் இருக்கும். இதனை தொல்காப்பியம், “நாய் துங்கினும் தாய் துஞ்சாது” என்பதால் அறியப்படும் (Puliyur Kesikan, 2013).

\section{தோழிக்காக வருந்துதல்}

தலைவிக்கு தோழி களவு வாழ்க்கையில் துணைபுரிந்தாலும் தலைவி தோழியை விட்டு பிரியமாட்டாள். தலைவி உடன் போக்கு போனபின் தோழி அவளை நினைத்து கண்கள் கலங்கின. தோழி கண்ணீர் சிந்துவதைக் கண்ட நற்றாய், “சொல்லிய முயலிப்” உடன்போக்கினை முன்னாலே தெரிவித்து தலைவியை தலைவனுக்குத் திருமணத்திற்கு உதவாமல் தவறிவிட்டாள் என தோழியை நொந்து நற்றாய், “தன்அமர் ஆயமொடு” வெளிப்படுத்துகிறாள் (Puliyur Kesikan, 2013).

\section{நற்றாயின் ஆற்றாமையும் பாசமும்}

செவிலித்தாய் நற்றாயிடம் தலைவியின் களவொழுக்கத்தை எடுத்துக்கூறி அறத்தோடு நிற்பாள். அந்நேரம் நற்றாய் தலைவியிடம் கூறி அவனுக்கே மணமுடிப்பதும் உண்டு. தலைவி தலைவனோடு உடன்போக்கும் நிகழும். இதில் நற்றாய் திரும்ப தலைவி வந்துவிடுவாள் என நம்பிக்கையுடன் காத்து இருப்பதை, "மறுவல் தூவிச் சிறுகரும்" எனக் காட்டுகிறார் (Puliyur Kesikan, 2013). நற்றாய் காக்கையைப் பார்த்து என் மகள் மீண்டு வரக்கரைவாய் எனக் கேட்பதன் மூலம் அவளுடைய ஆற்றாமைப் புலப்படுகிறது.

\section{சுற்றதார்க்குச் சொல்லியது}

உடன்போகிய தலைவி திரும்பி வந்த பிறகு தாய் உடன்போக்கு நிகழ்ந்த சூழலையும், அன்பில்லாத அறம் ஆகிய உடன்போக்கு நிகழ்வதையும் தலைவியின் கண்களை வர்ணித்து அவளின் அழகை இன்னும் மிகைப்படுத்தியும் தாம் பெருமையுறுவதை, “மாண்பில் கொள்கையொடு" எனும் பாடலில் விரிக்கிறார். தலைவன் தலைவியை யாரும் காணாத நேரத்தில் உடன்போக்கிற்கு அழைத்து செல்கிறான். பின் உரிய நேரம் பார்த்து தன் இல்லம் வந்து சேர்கிறான். வந்து பின் செய்ய வேண்டிய சடங்கை அவளுக்கு தலைவன் இல்லத்தில் செய்கின்றனர். சிலம்பு கழிக்கும் சடங்கை உங்கள் 
இல்லத்தில் செய்தீர்கள். பின் நடக்கும் வதுவை மணத்தையாவது எங்கள் வீட்டில் செய்யுங்கள் என்பதை, “நும்மனைச் சிலம்பு கழிஇய அயரினும், எம்மனை வதுவை நன்மணம் கழிகென” எனச் சொல்லி நற்றாய் புலம்புகின்றாள் (Puliyur Kesikan, 2020).

பின் ஊரார் பழிக்கு பயந்து உடன்போக்கு அறன் என்று சொன்னாலும் மனம் வேதனையடைந்து தலைவி திரும்பும் நாளை எண்ணி காத்திருக்கிறாள். தலைவி வந்ததை செவிலி மூலம் அறிந்ததும் மகிழ்ந்தும் அவள் உடன்போக்கிற்கான காரணத்தையும் சடங்கையும் முறைப்படி செய்ய வேண்டும் என்று நற்றாய் சுற்றத்தார்க்கு உரைக்கின்றாள்.

\section{செவிலித்தாயின் இயல்பும் துயரும்}

செவிலி தலைவன் தலைவியின் அன்பு வாழ்க்கை அறவாழ்க்கையாக மலருவதற்குப் பல்வேறு நிலைகளில் செயல்படுகிறாள். இவர்தம் கருத்தினால் சமூகச் சூழலோடு பொருந்தும் வகையில் அகவாழ்வில் மக்கள் உணர்வுகளைப் புரிந்து கொண்டு தலைவியின் உணர்வை அறிந்து அதற்கேற்ப செயல்படுகின்றாள்.

தலைவி தலைவனோடு உடன்போக்கில் சென்றுவிட்டாள். அவளைப் பேணி வளர்த்த செவிலித்தாய் உள்ளம் பெரிது வேதனைப்பட்டு வெதும்புகிறது. அவள் காதலைத் தான் புரியாமல் போன மடமையும் அவளை உடன்போக்கும் காத்துப் போவதை நிறுத்த இயலாமல் போன இயலாமையும் அவள் நெஞ்சை மேலும் காட்டுகிறது. அவள் உணர்வு மறுத்துப் புலம்பித் தளர்கின்றாள். அவள் துயரநிலை கண்டார். சிலர் அவருக்கு தேறுதல் உடைப்பவராகச் சிலர் சொல்ல அவள் அவர்களுக்கு தன்னையே வெறுத்துக் கூறுகிறாள். அத்த நீளிடை அவனொடு போகிய

முத்தோ வெண்பல் முகிழ்நகை மடவரல்

என அவரே கொடுத்த கூடமை செய்யும் பேற்றினைப் பெற்றனர் என்றாள் (Puliyur Kesikan, 2013). அவ்வாறு உடன் சென்ற தலைவனைத் தேடி பின் சென்ற செவிலித்தாய் எதிர் வருபவரைக் கேட்க அவளும் அவர்களைக் கண்டதனை உணர்ந்தனர். அதுகேட்ட அவள் அடிகள் எவ்வாறு அச்சுரத்திடையே நிலத்தில் பொருந்தினவே என்பதை, செய்வினைப் பொலிந்த செறிகழல் நோன்தாள் (389) மெல்லடி ஆற்றியது சொல்லோ என வருந்துகிறாள்.

கற்பக் காலத்தில் தலைவன் மணைக்கண் சென்று தலைவனும், தலைவியும் ஆவியும் உடலும் போல ஒருவரை ஒருவர் இன்றியமையாதவராய் அன்பால் கலந்து இன்புற்று வாழ்கின்ற நல்வாழ்க்கையின் மாண்பினைச் செவிலி கண்ணும் நெஞ்சம் குளிரக் கண்டு மீண்டும் அந்த மாண்பினை நற்றாய்க்குக் கூறி மகிழ்வித்தலையே பொருளாகக் கொண்டு குறிப்பிடப்பட்டுள்ளன. "மள்ளர் அன்ன மரவம் தமீஇ" செவிலியின் உண்மையான உள்ளத்தினை அறியமுடியும் (Puliyur Kesikan, 2013).

\section{முடிவுரை}

செவிலி தன் மகள் வாழ்வை நினைத்துப் பார்க்காமல் நற்றாயின் மகளை நினைத்து வருந்துவாள். தலைவிக்கு உண்மையான தாயாகவும், செவிலி தென்படுகிறாள். செவிலி தலைவியின் உள்ளம், செயல் ஆகியவற்றை அறிந்தவளாக தாயாகவே தென்பட்டும் தலைவியின் துன்பநிலையில் தானும் பங்கு கொண்டும் அக இலக்கியங்களில் தாய்மை நேயத்துடன் விளங்குகிறாள்.

\section{References}

Dakshinamurthy, A., (2004a) Sanga Ilakkiyam Ainkurunuru, New century book house, Chennai, India,

Dakshinamurthy, A., (2004b) Sanga Ilakkiyam Ainkurunuru, Volume 2, New century book house, Chennai, India, 
Ilankumaranar, U.A., (2018) Tolkappiyam Thelivurai, Fourth Tamil Sangam, Madurai, India.

Parimanam, A.M., Balasubramaniyam, K.V., (2004) Ainkurunuru, New century book house, Chennai, India

Puliyur Kesikan, (2013) Ainkurunuru (Kurinji, Palai), Sri Senbaga Pathppakam, Chennai, India.

Puliyur Kesikan, (2020) Ainkurunuru, Gowra Puthaga Maiyam, Chennai, India.

Sivalingan, A., (1995) Tolkappiyam Porulatikaram uraivalam, International Institute of Tamil Studies, Chennai, India.

\section{Funding}

No funding was received for conducting this study.

\section{Conflict of interest}

The Author has no conflicts of interest to declare that they are relevant to the content of this article.

\section{About the License}

(C) The Author 2022. The text of this article is open access and licensed under a Creative Commons Attribution 4.0 International License

\section{Cite this Article}

J. Jayavanisri, The Status of Women in Ainkurunuru Palai Thinai, Indian Journal of Multilingual Research and Development, Vol 3, Iss 1 (2022) 1-7. DOl: https://doi.org/10.54392/ijmrd2211 